\title{
Finite frequency inversion of cross-correlation amplitudes for ambient noise source directivity estimation
}

\author{
Arjun Datta ${ }^{1}$, Shravan Hanasoge ${ }^{1}$, and Jeroen Goudswaard ${ }^{2}$ \\ ${ }^{1}$ Department of Astronomy and Astrophysics, Tata Institute of Fundamental Research, Homi Bhabha \\ Road, Colaba, Mumbai 400005, India. \\ ${ }^{2}$ Shell India Markets Private Limited, Devanahalli Industrial Park, Mahadeva Kodigehalli, Bangalore 562 \\ 149, Karnataka, India.
}

Key Points:

- Seismic noise directivity estimation by waveform inversion of noise correlations

- Applied to an exploration scale data set

- Observational uncertainties incorporated in inversion for source distribution 


\begin{abstract}
We present a new method for determining the azimuthal variation of ambient noise sources, that combines the computational speed and simplicity of traditional approaches with the rigour of waveform-inversion-based approaches to noise-source estimation. This method is based on a previously developed theoretical framework of sensitivity kernels for crosscorrelation amplitudes. It performs a tomographic inversion for ambient noise sources on the Earth's surface and is suitable for small (local) scale studies. We apply the method to passive seismic data acquired in an exploration context, and account for azimuth-dependent uncertainties in observed cross-correlation amplitudes. Our inversion results correlate well with the azimuthal distribution of noise sources suggested by signal-to-noise ratio analysis of noise cross-correlation functions.
\end{abstract}

\title{
1 Introduction
}

The issue of heterogeneous noise sources or noise directivity has received significant attention in the field of ambient-noise seismology. Several studies have reported inaccuracies or limitations associated with the seismic measurements that can be extracted from noise cross-correlation functions (NCFs), such as surface wave dispersion (Froment et al., 2010; Kimman \& Trampert, 2010; Pedersen \& Krüger, 2007; Tsai, 2009; Wang, Luo, \& Yang, 2016; Yao \& van der Hilst, 2009) or anelastic attenuation (Stehly \& Boué, 2017; Tsai, 2011). Considerable effort has gone into developing tools for the proper exploitation of ambient-noise observations that are recognized to arise from anisotropically distributed sources (Curtis \& Halliday, 2010; Roux, 2009; Wang et al., 2016). Knowledge of the sources of ambient-field measurements, particularly their spatial and/or temporal variations, is now widely recognized as a prerequisite to any attempts to use these measurements to study Earth structure (e.g. Delaney et al., 2017; Lehujeur, Vergne, Maggi, \& Schmittbuhl, 2017).

The methods by which this information is typically obtained, with varying degrees of detail, fall under three broad categories: traditional array-processing or beamforming methods (e.g. Friedrich, Krüger, \& Klinge, 1998; Gal et al., 2014; Gerstoft \& Tanimoto, 2007; Liu et al., 2016; Löer, Riahi, \& Saenger, 2018; Nakata, Chang, Lawrence, \& Boué, 2015; Roux, Sabra, Gerstoft, Kuperman, \& Fehler, 2005; Ruigrok, Campman, \& Wapenaar, 2011), analysis of NCF asymmetry (Ermert, Villaseñor, \& Fichtner, 2016; Stehly, Campillo, \& Shapiro, 2006; Yang \& Ritzwoller, 2008) and more recently, inver- 
sions for ambient-noise sources (Delaney et al., 2017; Ermert, Sager, Afanasiev, Boehm, \& Fichtner, 2017; Lehujeur et al., 2017; Nishida \& Fukao, 2007). Inversions, by virtue of modelling and fitting waveforms (or attributes thereof) are the most rigorous, especially when based on spatially extended finite-frequency kernels.

The method we present in this paper carves a niche in this third category. Based on acoustic modelling and inverting only for noise-source directions, it is free of the need for expensive numerical simulations (Ermert et al., 2017) or ray-theoretical simplifications (Delaney et al., 2017). To the best of our knowledge it is also the first method to demonstrably account for uncertainties in observed NCF amplitudes. The simplifications of the method are that it is in 2-D (all sources and receivers required to be located on a plane) and structural variations remain unmodelled (a uniform wavespeed is assumed). At scales where the Earth's sphericity may be neglected, the former condition is not limiting because terrestrial seismic noise is known to be generated primarily on the Earth's surface (e.g. Webb, 1998). The latter restriction is also not a major impediment as amplitudes are used rather than traveltimes, so observed and synthetic waveforms can be reconciled by a judicious choice of measurement. However the restrictions do imply that our method is suitable for local-scale studies, not regional or continental scales (here the term local-scale refers to length scales that are short enough for the Earth's sphericity to be negligible). As emphasized by Lehujeur et al. (2017), local-scale studies are important candidates for noise directivity analysis, because small-aperture networks likely contain a significant fraction of measurements from interstation paths that are short compared to the seismic wavelength - measurements for which the effects of anisotropic noise sources are most pronounced (Bensen et al., 2007; Froment et al., 2010; Tsai, 2009).

We apply our method to an exploration-scale dataset acquired by Shell, which has captured azimuthally varying ambient seismic noise over a period of several months. The aim of this paper is method validation, not thorough characterization of our passive seismic data set, so we limit this study to a single frequency band. Applying the method to narrow frequency bands mitigates effects arising from neglecting (surface-wave) dispersion in the forward modelling. Our results are compared to those from NCF-asymmetry analyses. 


\section{Data and preliminary analysis}

Our data consist of one week (6-12 Nov 2016) of continuous ambient-field recordings taken by a network of 289 three-component geophones placed over an area $\sim 24 \mathrm{~km}$ $\times 28 \mathrm{~km}$ (Figure 1 ) and recording down to a lowest frequency of $\approx 0.1 \mathrm{~Hz}$. The data, stored in consecutive 10-minute intervals, have been downsampled from $4 \mathrm{~ms}$ to $20 \mathrm{~ms}$ sampling interval. For each 10-min window, vertical-component NCFs of $50 \mathrm{~s}$ duration are computed following well known processing techniques (Bensen et al., 2007), including running absolute-mean normalization and spectral whitening after filtering the raw data in the frequency band $0.1-0.5 \mathrm{~Hz}$. This frequency range was chosen in an attempt to include short-period microseismic noise (at $\approx 0.15 \mathrm{~Hz}$ ), though the observations contain negligible energy below $0.2 \mathrm{~Hz}$. Nonetheless, the chosen frequency band has high signal-to-noise ratio compared to the higher frequencies present in the data, and is narrow enough to produce waveforms without significant dispersion (see Figure S1). Cross correlations obtained as above for each 10-minute window, are averaged over a 24-hour period to obtain NCFs for each day. Clear travelling waves are observed in record sections of the NCFs (Figure S1).

We first attempt to characterize azimuthal variations in the strength of the daily NCFs. This is a type of NCF-asymmetry analysis that requires normalizing for different interstation distances (in each azimuth bin), since NCF amplitudes are expected to decay with distance (Cupillard \& Capdeville, 2010). Ignoring the effects of anelastic attenuation, one way to perform this normalization is to multiply observed amplitudes by the inter-receiver distance (e.g. Stehly et al., 2006). This formalism rests on the implicit assumption that the data exhibit the canonical geometrical spreading rate of surface waves in a laterally homogeneous medium. We test this assumption in our data and find that there is a range of azimuths for which the observed NCF energies $(E)$ are poorly described by the canonical decay rate. Sorting our daily NCFs into azimuth bins of $4^{\circ}$, regardless of absolute receiver location and inter-receiver distance $r$, we fit $1 / r$ curves to the observed scatter in NCF energies plotted as a function of $r$ (Figure S2). Each NCF yields measurements for two (radially opposite) directions, because energies in the positive and negative branches of the waveform are measured separately. Figure 2 shows a polar plot of the $\chi^{2}$ misfit comprising deviations from the $1 / r$ approximation, for each azimuth bin. Given the significant deviations from theory at some azimuths, we infer that a normalization such as that of Stehly et al. (2006) would produce biases and lead to erroneous 
interpretation of dominant source directions. The method we propose in this paper is free of this potential bias because it does not require presuming an amplitude decay rate, but instead uses the observed amplitudes to quantify data errors in an inverse problem.

Finally, we recognize that the amplitudes we work with are a function of the preprocessing applied to the raw noise recordings. However this does not mean that our use of amplitudes is either tenuous or unusual. The ambient noise literature contains several examples of studies that employ similar processing to ours and exploit correlation amplitudes. Notable among these are those that measure Rayleigh wave ellipticity or $\mathrm{H} / \mathrm{V}$ amplitude-ratio (Lin, Tsai, \& Schmandt, 2014; Muir \& Tsai, 2017), requiring care to ensure that relative amplitudes between different component correlations are preserved through the pre-processing. In our case, as long as it is consistent across the receiver network, the particular pre-processing choice is not important because absolute amplitude values are irrelevant. Absolute values simply scale with the strength of noise sources used to forward model the NCFs. Only the relative amplitudes between receivers, and between positive and negative branch correlations, are decisive in unraveling noise directionality by our method. This will become clear in the next section.

\section{Methodology and synthetic tests}

Hanasoge (2013) introduced sensitivity kernels for NCF amplitudes, based on analytical solutions to an acoustic-wave equation in homogeneous 2-D media. In this method, which we briefly recap, forward modelling rests on a closed form expression for the medium Green's function:

$$
G\left(\mathbf{x}, \mathbf{x}^{\prime}, \omega\right)=H_{0}^{(1)}\left(\frac{\omega}{c}\left|\mathbf{x}-\mathbf{x}^{\prime}\right|\right),
$$

where $c$ is the uniform medium wavespeed, $H_{0}^{(1)}$ is the Hankel function of the first kind, and other symbols have their usual meanings. NCFs are thus modelled using a power spectrum $P(\omega)$ of spatially uncorrelated noise sources and their spatial-amplitude distribution $s(\mathbf{x})$ in the 2 -D plane. The "ensemble cross correlation" (Tromp, Luo, Hanasoge, \& Peter, 2010) between two receiver locations $\mathbf{x}_{\alpha}$ and $\mathbf{x}_{\beta}$, is computed as:

$$
\left\langle C_{\alpha \beta}\right\rangle=\int H_{0}^{(1) *}\left(\frac{\omega}{c} \Delta_{\alpha}\right) H_{0}^{(1)}\left(\frac{\omega}{c} \Delta_{\beta}\right) P(\omega) s(\mathbf{x}) d^{2} \mathbf{x},
$$

where $\Delta_{\alpha}=\left|\mathbf{x}_{\alpha}-\mathbf{x}\right|$ is introduced for brevity. To construct sensitivity kernels, the method uses a measurement which is the NCF energy (denoted by $A$ ) in a time window 
of interest $(w)$, either on the positive or negative correlation branch:

$$
A_{\alpha \beta}=\sqrt{\int w(t)\left\langle C_{\alpha \beta}(t)\right\rangle^{2} d t} .
$$

Source kernels, which define the sensitivity of this measurement to $s(\mathbf{x})$ and hence encapsulate the physics of the problem, follow from variational principles and are given by:

$$
K_{\alpha \beta}(\mathbf{x})=\frac{1}{2 \pi}\left(\frac{1}{A_{\alpha \beta}^{\text {syn }}}\right)^{2} \int H_{0}^{(1) *}\left(\frac{\omega}{c} \Delta_{\alpha}\right) H_{0}^{(1)}\left(\frac{\omega}{c} \Delta_{\beta}\right) P(\omega) C_{\alpha \beta}^{w}(\omega) d \omega,
$$

where $C_{\alpha \beta}^{w}$ denotes the windowed cross-correlation, and the superscript on $A$ denotes measurements made on the synthetic NCFs. The same measurement is made on the observed NCFs in order to solve the inverse problem.

Given a network of receivers, this method lends itself to a fast Hessian-based tomographic inversion for source distribution. A misfit functional is defined which penalizes the logarithmic ratio of $A^{\text {obs }}$ to $A^{\text {syn }}$ for all receiver pairs (A.2), and a misfit kernel $K$ is obtained as the sum of individual source kernels weighted by their misfits (A.6). Except for details of the measurement and misfit, this is analogous to, for instance, classical (earthquake-based) finite frequency traveltime tomography with banana-doughnut kernels (e.g. Dahlen, Hung, \& Nolet, 2000).

Hanasoge (2013) used synthetic tests to show the suitability of the misfit kernels for tomographic inversion. In this study, we take the straightforward next step of actually performing these inversions. We choose a modelling domain encompassing the receiver network, with a uniform grid spacing of $0.25 \mathrm{~km}$ and a uniform wavespeed of 2.1 $\mathrm{km} / \mathrm{s}$, ensuring a sampling of 16 grid points per shortest wavelength $(\approx 4.4 \mathrm{~km})$. The chosen wavespeed is estimated from the real data by picking the maxima of envelopes on NCF record sections (e.g. Figure S1) and averaging positive and negative branch values. Since we work with amplitudes, there is a trade-off between the strength of sources and their distances from the receiver network, which we do not endeavour to resolve. We aim only to constrain the relative azimuthal distribution of noise sources. To this end, we parameterize our spatial source distribution as a ring of sources surrounding the network (see Figure 3a). The ring comprises $M$ spatial Gaussians centered every $10^{\circ}$ (which form a basis set for model expansion) and has a radius large enough to enclose the network. In this paper we present results for a ring of radius $25 \mathrm{~km}$, but have verified with synthetic tests as well as real data, that the size of the ring has a negligible impact on the inferred source directions as long as it is wider than the array aperture. The model 
vector, of length $M$, simply contains the basis-function coefficients. We choose $M=36$, thereby working with a highly tractable 36 -parameter model space. Since individual kernels for each receiver pair are computed, the gradient and Hessian of the misfit are both available, allowing us to invert using a standard quasi-Newton scheme (see Appendix A). The model update is obtained separately for positive- and negative-branch measurements and the two results are averaged.

Our inverse method is validated by a series of synthetic tests, performed using the same network configuration as the real dataset. We begin by using a test model that follows the parameterization used for the inverse problem (Figure 3), so all sources are constrained to lie on the same ring around the receiver network. We run a series of examples using different-size subsets of the complete receiver network and the two end-member cases are shown in Figure 3. The combination of misfit kernels from positive- and negativebranch measurements illuminates all model perturbations and the inversion recovers the true model with an accuracy that improves, as expected, with increased network coverage. With 256 receivers, the test results are essentially perfect — all source locations and amplitudes are recovered exactly. Next, we employ a test model that is free of the aforementioned parameterization, i.e. it contains arbitrarily oriented sources at arbitrary distances from the receiver network (Figure 4). In this case it is difficult to quantify the accuracy of the model obtained by inversion (true source amplitude information is lost), but we argue that source directions and their relative strengths are recovered reasonably well. Taken together, results from these tests validate the inverse algorithm and the imaging concept advocated by this paper. In particular, the latter tests justify our choice of $50 \mathrm{~km}$ for the size of the ring with which we parameterize the model space. In order to justify the size of the computational domain used in the next section $(60 \mathrm{~km} \times 60 \mathrm{~km})$ and in recognition of the fact that ambient noise propagation is not necessarily local, we run similar tests for the case where the actual sources lie outside the domain used for inverse modelling. These are shown in Appendix B and also produce satisfactory results.

\section{Application to real data}

We apply the method to our data after tapering and downsampling the observed NCFs to $0.2 \mathrm{~s}$. This factor-of-10 downsampling is in accord with the highest frequency considered $(0.5 \mathrm{~Hz})$ and reduces the computational cost of forward modelling. Based on the work of Section 3, the modified NCFs are subjected to inversion using a $60 \mathrm{~km} \times$ 
$60 \mathrm{~km}$ modelling domain and 256 receivers. However three important considerations remain: the choice of $P(\omega)$ for the noise sources, the choice of window size for the measurements $A$, and quantification of observational errors or uncertainties. We discuss each one in detail.

A judicious choice of $P(\omega)$ is required to produce synthetic NCFs that match the input observed NCFs not only in shape (frequency content) but also - in order to initialize the inversion - in order of magnitude of amplitude. Since $P(\omega)$ contributes to the synthetic NCFs through interaction with other terms in a spatial integral (Hanasoge, 2013, eq. 11), it is empirically estimated as a skew-symmetric Gaussian function (Figure S3a). Its amplitude is determined by requiring that the initial synthetics have energies equal to an average representation of the observed NCF energies, namely the $1 / r$ curve that best fits the observed NCF energies as a function of interstation distance (Figure S3b). This is similar to the analysis of Section 2 but here, the energies are computed for complete NCFs (not separately for positive and negative branches) and all observations are taken together, regardless of azimuth. The second consideration, that of the measurement window, is important because the window needs to be narrow enough to ensure that it fits only the coherent signals in the NCFs and yet broad enough to accommodate those cases where the signals in the observed and synthetic NCFs are significantly offset from each other in time (the chosen wavespeed is likely a poor approximation in some parts of the study region). We set an $8 \mathrm{~s}$ window around the arrival time dictated by the chosen homogeneous wavespeed. The taper applied to the observed NCFs exists only between the outer edge of this measurement window and the waveform extremity.

Lastly, the data analysis is made meaningful by introducing data errors into the inverse problem. We consider two types of error: $\sigma_{1}$, based on the energy-decay analysis of Section 2, and $\sigma_{2}$, due to the signal-to-noise ratio (SNR) of the observed NCFs. The first error, $\sigma_{1}$, is simply an estimate for $\Delta A$ obtained using $A=\sqrt{E}$, and $\Delta E$ as yielded by curve-fitting in the relevant azimuth bin (e.g. Figure S2). SNR is defined as the ratio of the energy density of an NCF in a time window of interest (we use the inversion measurement window), to its energy density outside that window. Similar to the analysis of Section 2, SNR is measured separately on the positive and negative branches, yielding two values for each $\mathrm{NCF}$ and therefore an error estimate $\left(\sigma_{2}\right)$ for each measurement made for source inversion. SNR values are translated into data errors by qualitatively identifying three SNR regimes $(>3,2<$ SNR $<3$ and $<2)$ to which we as- 
sign $\sigma_{2}=5 \%, 50 \%$ and $80 \%$ respectively of the $A$ value. We assume that errors on different measurements are uncorrelated $\left(\mathbf{C}_{D}\right.$ in (A.12) is diagonal) and that the two errors considered are independent, i.e. $\sigma^{2}=\sigma_{1}^{2}+\sigma_{2}^{2}$. The latter assumption is based on the observation that high SNR values coincide with large departures from a $1 / r$ energy decay rate (see Figures 2,6). Other sources of error, such as neglecting the topography of the region and differing station elevations, are not explicitly accounted for.

The inverse algorithm thus customized, is applied separately to the observed NCFs for each day. We achieve total misfit reductions of $\sim 70-80 \%$, with at least $50 \%$ of measurements being fit to an accuracy greater than $70 \%$. An example of this quantitative summary for one of the days is shown in Figure 5. Finally, we corroborate our results with the simple idea of using SNR itself as a proxy for source directionality. Azimuthal polar plots of SNR are obtained in a manner similar to Figure 2, with the exception that, here, distance does not play an explicit role - SNR values for different interstation distances within an azimuth bin are averaged. Yang and Ritzwoller (2008) used this kind of analysis on long-range correlations to infer noise-source directions at continental scales. In this study, we find that subtle variations - on daily time scales - in the dominant wave-propagation directions (primarily between ENE and NNE) inferred from SNR polar plots, correlate well with variations in the azimuthal noise-source distribution obtained by our inverse method (Figure 6). Also, there is qualitative agreement between the two methods, on the apparent strength of noise sources on different days - SNR values are highest on days 3-5, as are source amplitudes obtained by inversion.

\section{Conclusions and Discussion}

We have introduced a new method of determining ambient noise directionality by inverting NCF amplitudes. In its present form, the method is suitable for local-scale studies, where the effects of Earth's sphericity are negligible. The main attraction of the technique compared to existing approaches is the combination of computational ease and rigorous waveform-fitting. Although the method owes its economy to strong simplifying approximations, these do not preclude its application. In particular, the use of amplitudes ensures that Earth structure has a diminished impact on the measurements. We applied the technique to a study region with known strong lateral variations in geological structure, and achieved acceptable waveform fits in the inverse modelling. The particular model parameterization used in this study is in line with the expectation of distant noise sources 
in our region of study, but we observe that other parameterizations may just as well be used without altering the technique fundamentally. The "ideal" choice of parameterization may be problem-dependent; it should be guided by prior knowledge (e.g. strong local sources) and computational expediency.

Our real data application systematically accounts for observational uncertainties, and demonstrates the suitability of the method for studying non-stationarity in ambientnoise-source distributions over short timescales (e.g. hours or days for reservoir monitoring). This is possible because the technique may be applied to ambient seismic noise of shorter duration than is typically used in most applications. In this respect, it contributes to a growing body of seismological research (e.g. Fichtner, Stehly, Ermert, \& Boehm, 2017; Roux \& Ben-Zion, 2017) that treats NCFs as fundamental seismic observables in their own right, without the requirement of convergence to an interstation Green's function. On that note we point out that when inverting for both sources and structure (as is characteristic in noise interferometry without Green's function retrieval), the technique presented in this paper may be used to constrain sources in the first step of the coupled inverse problem solution, where structure is assumed to be uniform.

Lastly, in this study we have ignored potential biases resulting from the fact that data processing is not accounted for in the forward modelling; in other words the observed and synthetic waveforms are not processed in the same way. Theoretical work to address this issue has already emerged (Fichtner et al., 2017) and while we acknowledge it is an important consideration for the future, we do not believe it to be a significant problem for the data analyzed in this paper. The results obtained (Figure 6), suggest dominant noise sources to the South West of the receiver network, which is consistent with its geographical location. 


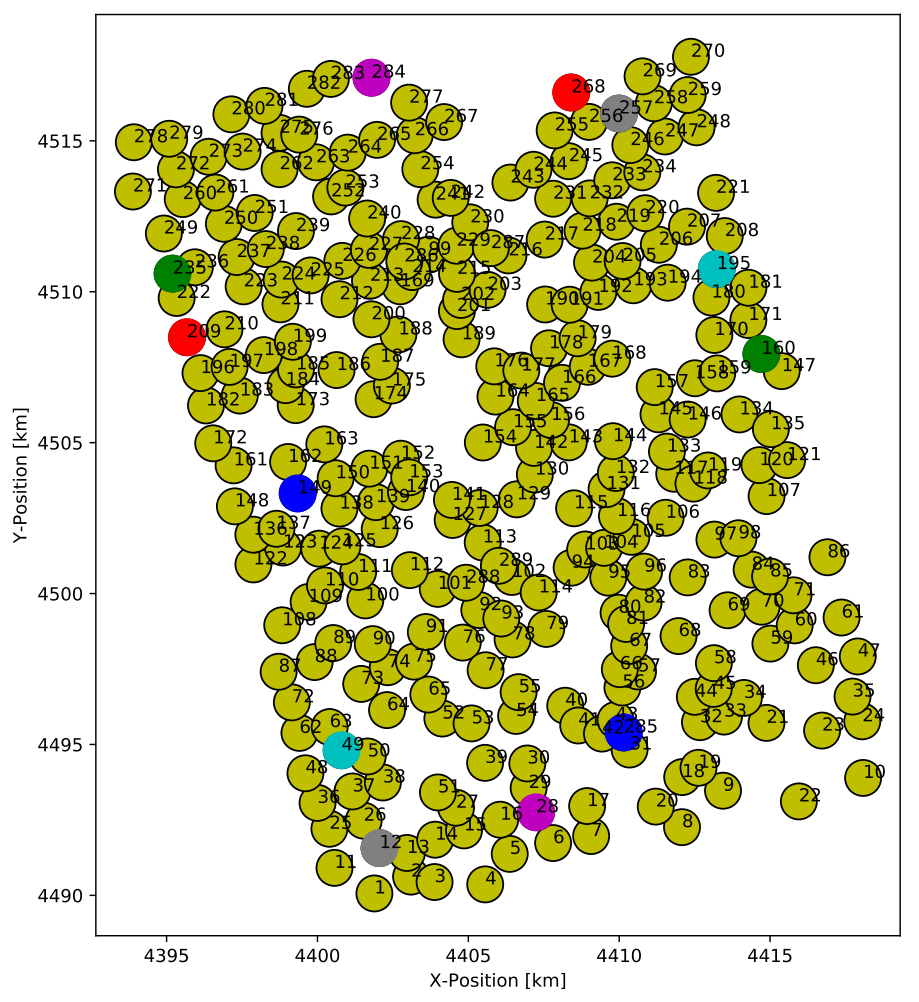

Figure 1. Map view of network of recording geophones, numbered from 1 to 289. Colours other than yellow indicate six receiver pairs selected for later reference in Figure 5. 


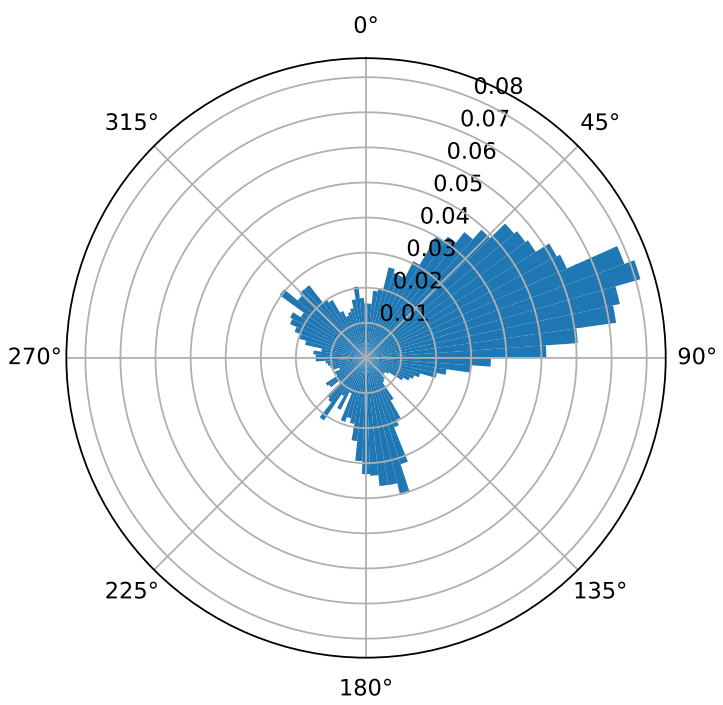

Figure 2. Single-day example (day 3 ) of azimuthal variation in the departure of the observed cross-correlation energies from a $1 / r$ energy decay regime, where $r$ is interstation distance. Plotted values are the $\chi^{2}$ misfit obtained in each azimuth bin - refer to text and Figure S2. 
(a)
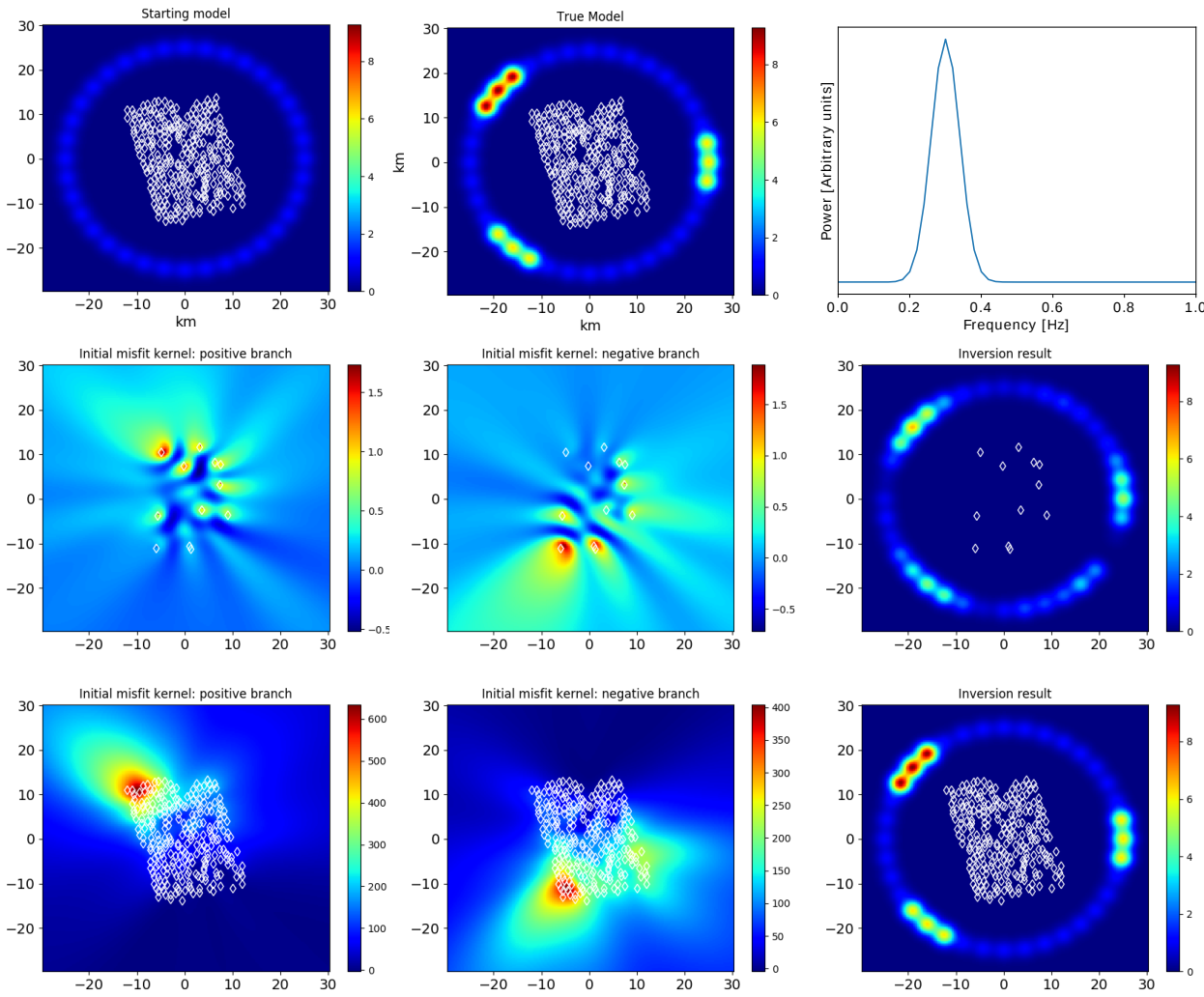

Figure 3. Synthetic test with test model following the parameterization used for the inverse

problem. (a) Left: Reference model $(60 \times 60 \mathrm{~km})$ with an azimuthally homogeneous $s(\mathbf{x})$ shown in colour. All basis functions have unit amplitude in this model and it serves as the starting model for inversions. Middle: The "true model" for the synthetic test, comprising three highamplitude regions relative to the reference model. Right: Assumed $P(\omega)$ for the sources, centered at $0.3 \mathrm{~Hz}$. (b) Inversion performed using only 12 receivers: starting misfit kernels for the positive(left column) and negative-branch (middle column) measurements, and the inversion result after 8 iterations (right column). (c) Same as (b) but with 256 receivers. Note that all the model plots have the same colour scale and in all plots, the white diamonds mark the receiver locations. 
(a)
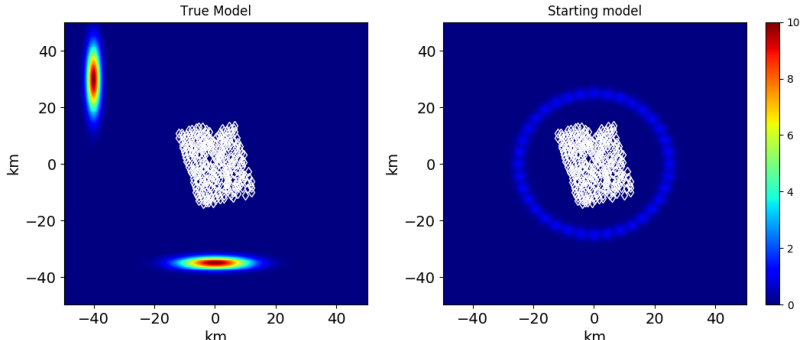

(b)
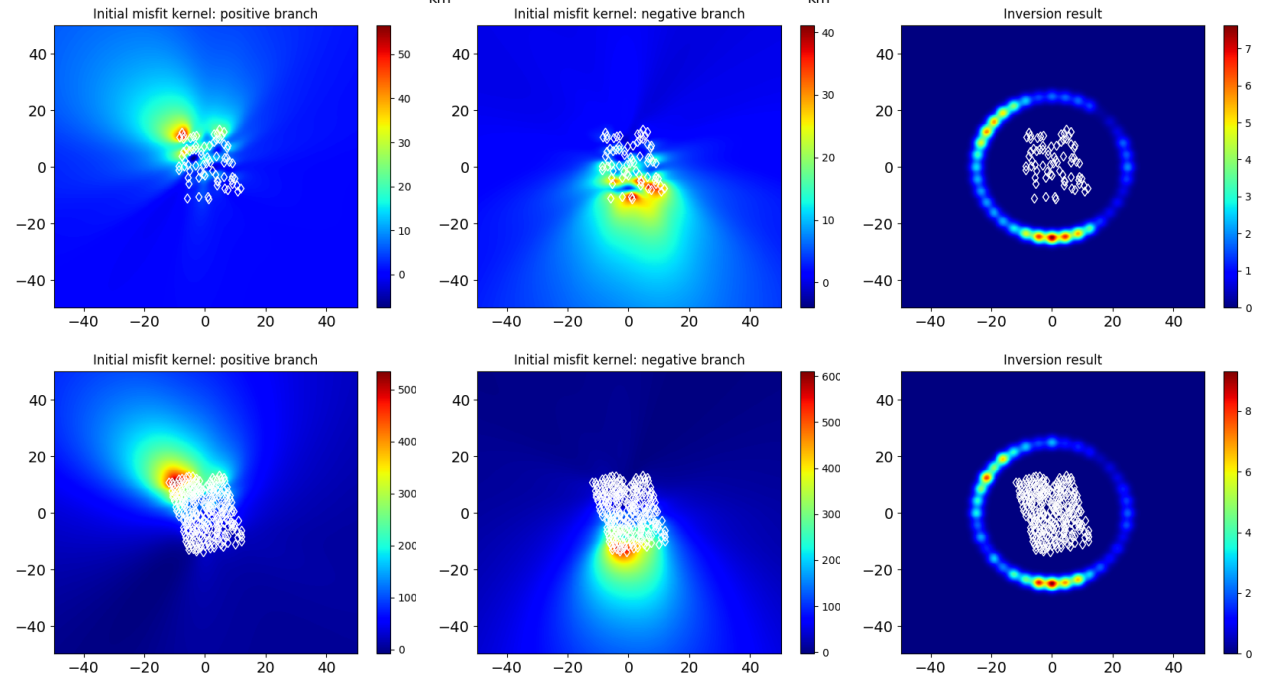

Figure 4. Synthetic test with test model not parameterized as in the inverse problem. (a) True model (left) and starting model for inversions (right, same as in Figure 3). Note the larger modelling domain $(100 \times 100 \mathrm{~km})$ compared to Figure 3 . In the true model, the high amplitude regions to the North West and South of the receiver network are at distances of $50 \mathrm{~km}$ and 35 $\mathrm{km}$ respectively, from the network centre. (b)-(c) same as in Figure 3 except that (b) uses 72 receivers. Both inversions predict higher source amplitudes South of the receiver network than North West of it (although the difference is subtle and hard to discern visually); this is commensurate with the sources in the true model being closer to the network in the South than in the North West. 
(a)

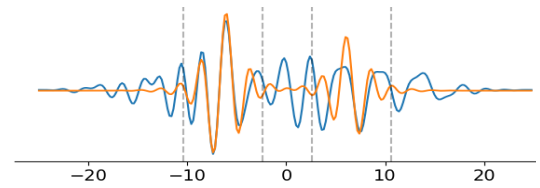

(c)

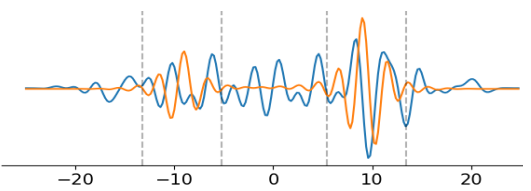

(e)

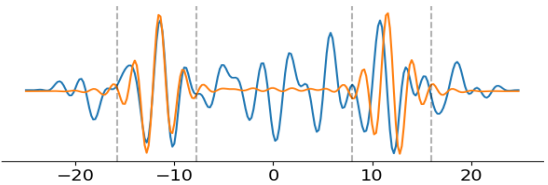

(g)

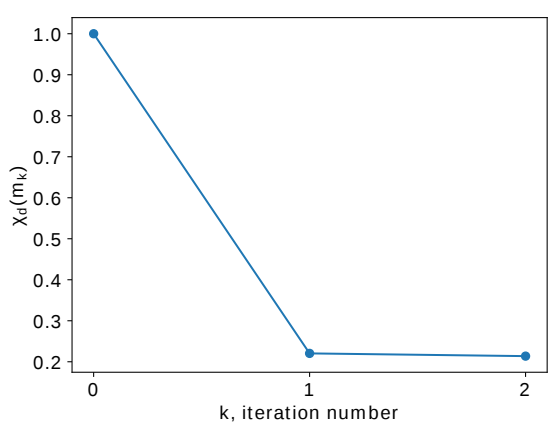

(b)

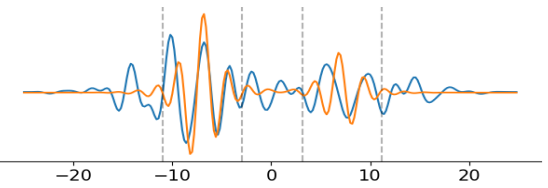

(d)

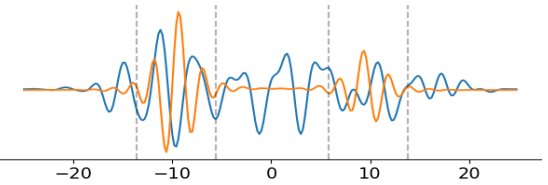

(f)

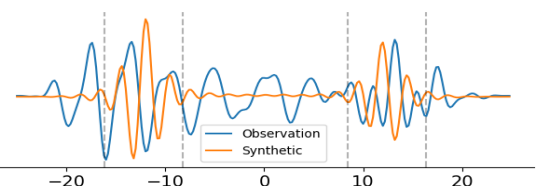

(h)

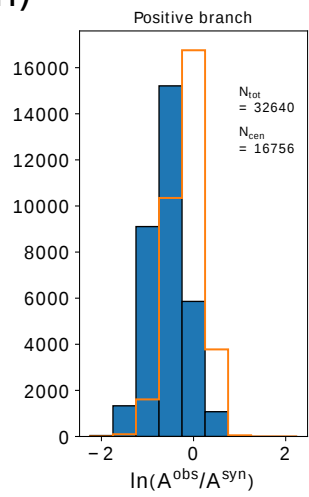

Figure 5. Summary of inversion for day 3. (a)-(f) Six examples of waveform fits after inversion; these correspond to receiver pairs highlighted in Figure 1, in blue (285-149), red (268-209), green (235-160), cyan (195-49), magenta (284-28) and grey (257-12) respectively. All plots share the same legend and the vertical black dashed lines represent the 8-s measurement window in each case. Note that (e) has the greatest symmetry in amplitude between the positive and negative branches, and it corresponds to a receiver pair that is oriented approximately transverse to the dominant incoming energy direction (see Figure 6). (g) Evolution of the total data misfit $\chi_{d}$ (normalized for plotting) through the inversion, showing a misfit reduction of nearly $80 \%(\mathrm{~h})$ Histograms of $\Delta d$ values from all interstation pairs in the starting model (blue filled bars) and in the final inverted model (orange step bars). Note that data errors are included in $\chi_{d}$ but not in $\Delta d$. Text on the plots indicates the total number of measurements $\left(N_{t o t}\right)$ and those that lie in the central histogram bin after inversion $\left(N_{c e n}\right)$. The central bin corresponds to $|\Delta d|<=0.25$, or a maximum waveform discrepancy (as defined by the measurement) of about $28 \%$. In this case, 32829 measurements (out of the total 65280) lie in the central bin after inversion, meaning that $\sim 50.3 \%$ of total measurements are fit to an accuracy of at least $72 \%$. 

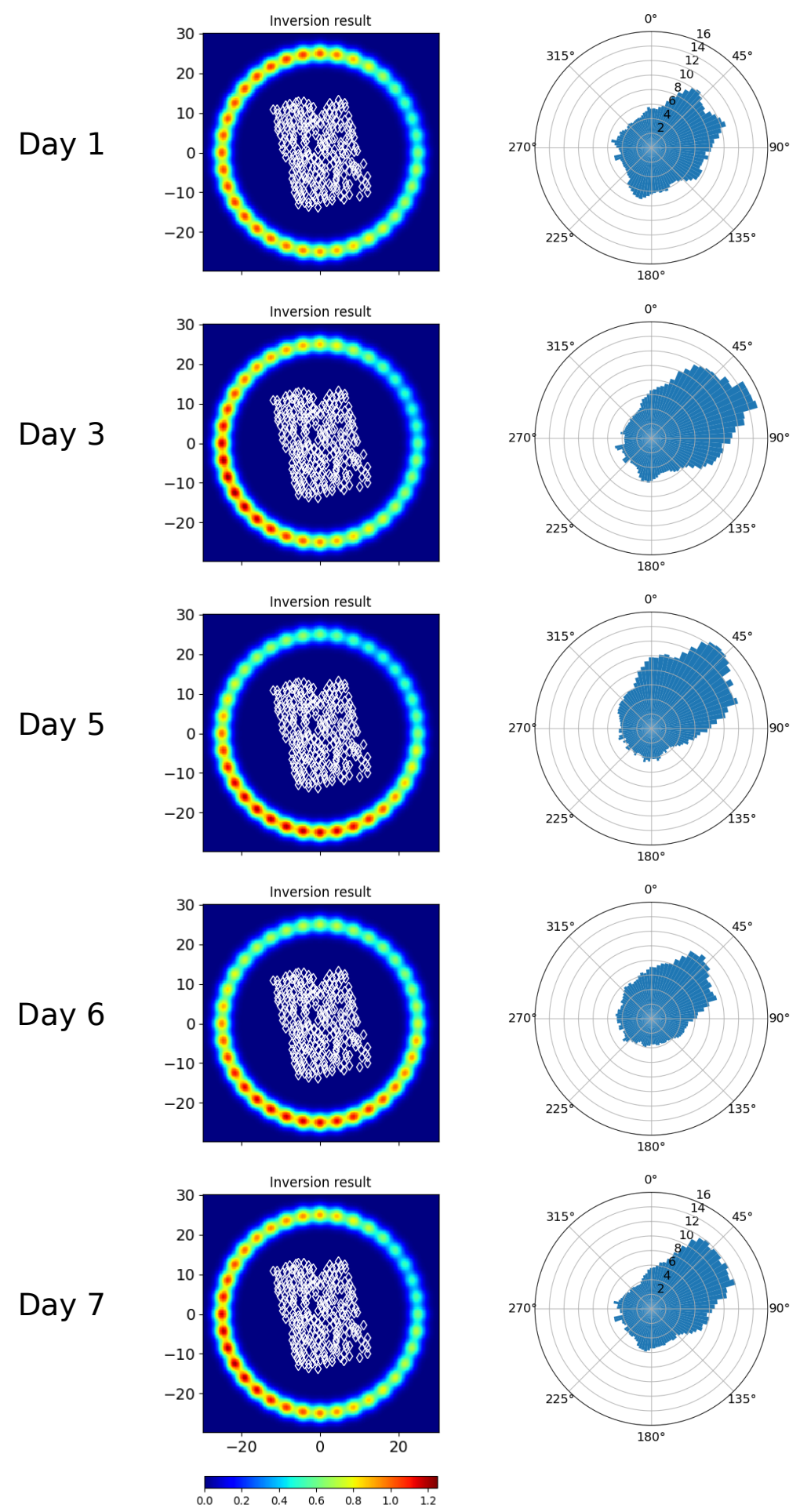

Figure 6. Results corresponding to different days of data from our method (left column) and from SNR analysis (right column). In the SNR plots, each bar points in the direction of wave propagation, i.e. it points away from the source location. 


$$
\delta \chi=-\int K(\mathbf{x}) \delta s(\mathbf{x}) d^{2} \mathbf{x}
$$

where $s(\mathbf{x})$ is the spatial source distribution, $K(\mathbf{x})$ the misfit kernel and $\chi$ the misfit functional defined as:

$$
\begin{aligned}
\chi & =\frac{1}{2} \sum_{i}\left(\ln \frac{A_{i}^{\text {obs }}}{A_{i}^{\text {syn }}}\right)^{2} \\
& =\frac{1}{2}\left[\mathbf{d}_{\text {obs }}-\mathbf{d}_{\text {syn }}\right]^{T}\left[\mathbf{d}_{\text {obs }}-\mathbf{d}_{\text {syn }}\right]
\end{aligned}
$$

with index $i$ denoting the $i^{\text {th }}$ receiver-receiver pair. Expanding the model perturbation into the model-space basis functions $B_{j}$, we have

$$
\delta s(\mathbf{x})=\sum_{j=1}^{M} \delta m_{j} B_{j}(\mathbf{x})
$$

Using (A.3) in (A.1) gives

$$
\delta \chi=\sum_{j=1}^{M}\left[-\int K(\mathbf{x}) B_{j}(\mathbf{x}) d^{2} \mathbf{x}\right] \delta m_{j}
$$

from which it is clear that the quantity in square brackets represents the gradient (g)

of $\chi$ :

$$
\frac{\partial \chi}{\partial m_{j}}=-\int K(\mathbf{x}) B_{j}(\mathbf{x}) d^{2} \mathbf{x}=g_{j}
$$

To express the gradient in terms of the customary $\mathbf{G}$ matrix that linearizes the inverse problem, we need only invoke the fact that the misfit kernel is a weighted sum of source kernels for individual receiver pairs (Hanasoge, 2013, eq. 30):

$$
K=\sum_{i} \ln \left(\frac{A_{i}^{\text {obs }}}{A_{i}^{\text {syn }}}\right) K_{i}(\mathbf{x})
$$

Using (A.6) in (A.5), we have

$$
\begin{aligned}
g_{j} & =-\sum_{i} \ln \left(\frac{A_{i}^{\text {obs }}}{A_{i}^{\text {syn }}}\right) \int K_{i}(\mathbf{x}) B_{j}(\mathbf{x}) d^{2} \mathbf{x} \\
& =-G_{i j} \Delta d_{i}
\end{aligned}
$$

or

$$
\mathrm{g}=-\mathbf{G}^{T} \Delta \mathbf{d}
$$


where the $\mathbf{G}$ matrix and $\Delta \mathbf{d}$ vector are given by:

$$
\begin{aligned}
G_{i j} & =\int K_{i}(\mathbf{x}) B_{j}(\mathbf{x}) d^{2} \mathbf{x} \\
\Delta d_{i} & =\ln \left(\frac{A_{i}^{\mathrm{obs}}}{A_{i}^{\mathrm{syn}}}\right)
\end{aligned}
$$

Using $\mathbf{G}$, the approximate Hessian $\mathbf{H}$ for a Gauss-Newton inversion is easily computed:

$$
\mathbf{H}=\mathbf{G}^{T} \mathbf{G}
$$

In practice, the canonical expressions (A.8) and (A.10) are modified, because the objective function itself is modified by damping (required to overcome solution underdeterminacy) and weighting by model or data (co-)variances (required to control the extent of damping or to incorporate observation errors, as in Section 4). The inverse problem we actually work with is (e.g. Tarantola, 2005):

$$
\begin{aligned}
\tilde{\chi} & =\frac{1}{2}\left[\mathbf{d}_{\text {obs }}-\mathbf{d}_{\text {syn }}\right]^{T} \mathbf{C}_{D}^{-1}\left[\mathbf{d}_{\text {obs }}-\mathbf{d}_{\text {syn }}\right]+\frac{1}{2}\left[\mathbf{m}-\mathbf{m}_{\text {prior }}\right]^{T} \mathbf{C}_{M}^{-1}\left[\mathbf{m}-\mathbf{m}_{\text {prior }}\right] \\
& =\chi_{d}+\chi_{m} \\
\tilde{\mathbf{g}} & =-\mathbf{G}^{T} \mathbf{C}_{D}^{-1} \Delta \mathbf{d}-\mathbf{C}_{M}^{-1}\left(\mathbf{m}-\mathbf{m}_{\text {prior }}\right) \\
\tilde{\mathbf{H}} & =\mathbf{G}^{T} \mathbf{C}_{D}^{-1} \mathbf{G}+\mathbf{C}_{M}^{-1}
\end{aligned}
$$

Here $\mathbf{C}_{D}$ and $\mathbf{C}_{M}$ are the model and data covariance matrices. We use $\mathbf{C}_{D}=\mathbf{I}$ and $\mathbf{C}_{D}(i, j)=\sigma_{i j}^{2} \delta_{i j}$ in sections 3 and 4 respectively. Correspondingly, for $\mathbf{C}_{M}$ we use $\mathbf{C}_{M}=$ $\frac{1}{\gamma^{2}} \mathbf{I}$ and $\mathbf{C}_{M}(i, j)=\frac{1}{\gamma^{2}} \exp \left(\frac{-(i-j)^{2}}{2 L^{2}}\right)$ respectively. Here $\gamma$ is a damping parameter and $L$ is a correlation length that controls the smoothness of the model; they are both determined empirically. These choices for $\mathbf{C}_{D}$ and $\mathbf{C}_{M}$ imply that the synthetic test inversions are carried out with no data errors and no smoothing, whereas the real data inversions are done with both errors and smoothing included.

Choosing $\mathbf{m}_{\text {prior }}=\mathbf{m}_{0}$ and applying the Gauss-Newton method $\tilde{\mathbf{H}} \Delta \mathbf{m}=-\tilde{\mathbf{g}}$ to (A.11) leads to the following iterative solution:

$$
\mathbf{m}_{k+1}=\mathbf{m}_{k}+\left[\mathbf{G}_{k}^{T} \mathbf{C}_{D}^{-1} \mathbf{G}_{k}+\mathbf{C}_{M}^{-1}\right]^{-1}\left[\mathbf{G}_{k}^{T} \mathbf{C}_{D}^{-1} \Delta \mathbf{d}_{k}+\mathbf{C}_{M}^{-1}\left(\mathbf{m}_{k}-\mathbf{m}_{0}\right)\right]
$$

where index $k$ denotes the iteration number. 
B Additional synthetic tests 
(a)
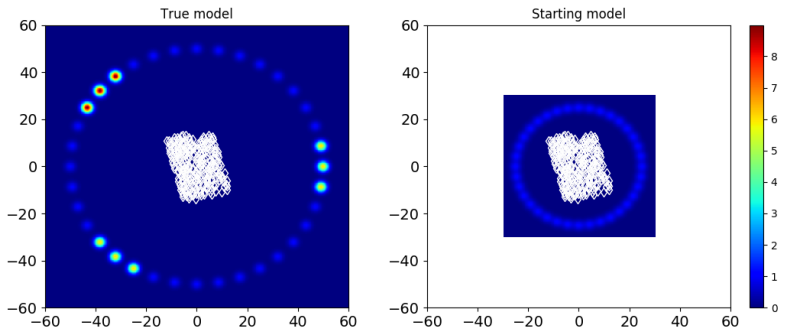

(b)
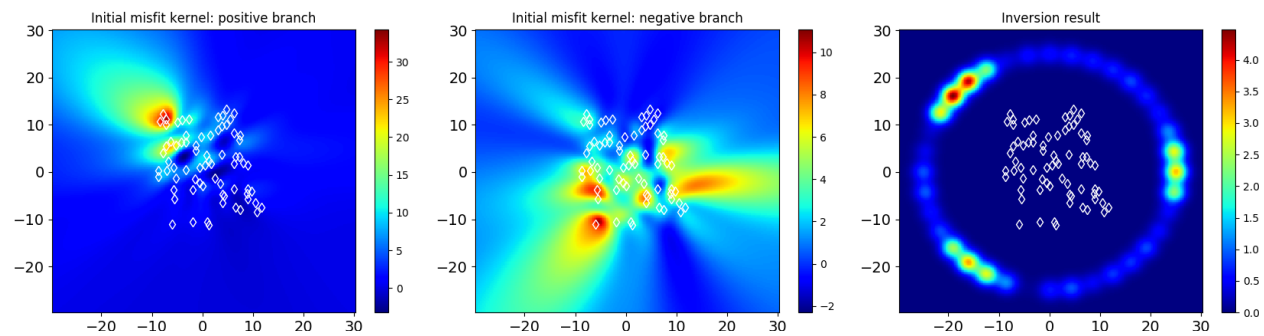

(c)
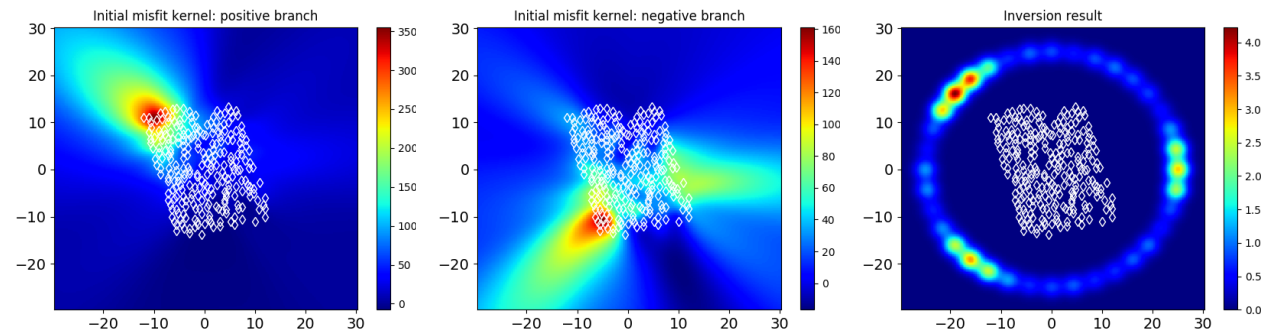

Figure B.1. Synthetic test with test model following a similar parameterization as in the inverse problem, but with sources lying outside the inverse modelling domain. (a) $120 \times 120 \mathrm{~km}$ true model (left) and $60 \times 60 \mathrm{~km}$ starting model for inversions (right). The ring of sources in the true model has a $50 \mathrm{~km}$ radius, while the starting model is exactly the same as in Figure 3. (b) Inversion performed using 72 receivers: starting misfit kernels for the positive- (left column) and negative-branch (middle column) measurements, and the final inversion result (right column). Note the larger scale of the plots compared to part (a), as only the inverse modelling area is shown. Also note lower source amplitudes in the inverted model as compared to the true model, given the smaller ring (radius $25 \mathrm{~km}$ ) and hence nearer sources in the inverted model. (c) Same as (b) but with 256 receivers. 
(a)

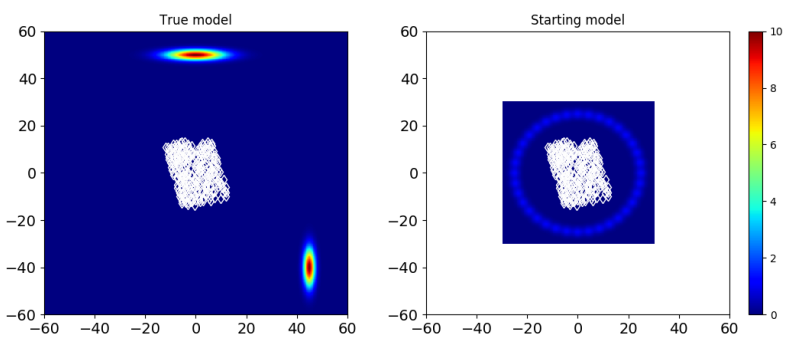

(b)
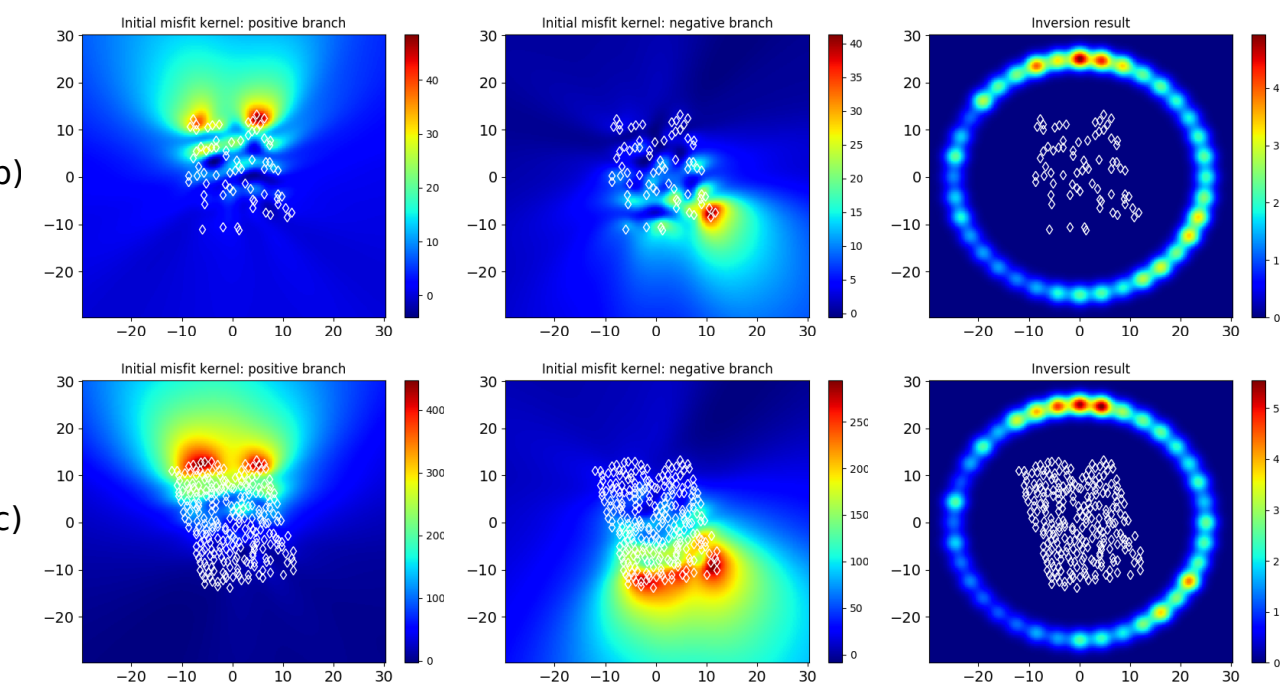

Figure B.2. Synthetic test with test model not parameterized as in the inverse problem and with sources lying outside the inverse modelling domain. (a) - (c) Same as Figure B.1. 


\section{Acknowledgments}

This work was funded by Shell India Markets Pvt. Ltd. Computing was performed on the SEISMO cluster at Tata Institute of Fundamental Research. The passive seismic data used in this study are owned by Shell International Exploration and Production B.V. They were collected for commercial purposes. These data and the permission to use them, for non-commercial purposes only, can be obtained from Shell (https://www.shell.in/aboutus/contact-us.html). We thank Laura Ermert and an anonymous reviewer for their comments and suggestions which helped to improve this manuscript.

\section{References}

Bensen, G. D., Ritzwoller, M. H., Barmin, M. P., Levshin, A. L., Lin, F., Moschetti, M. P., .. Yang, Y. (2007). Processing seismic ambient noise data to obtain reliable broad-band surface wave dispersion measurements. Geophys. J. Int., 169(3), 1239-1260. doi: 10.1111/j.1365-246X.2007.03374.x

Cupillard, P., \& Capdeville, Y. (2010). On the amplitude of surface waves obtained by noise correlation and the capability to recover the attenuation: a numerical approach. Geophys. J. Int.. doi: 10.1111/j.1365-246X.2010.04586.x

Curtis, A., \& Halliday, D. (2010). Directional balancing for seismic and general wavefield interferometry. GEOPHYSICS, 75(1), SA1-SA14. doi: 10.1190/1 .3298736

Dahlen, F. A., Hung, S.-H., \& Nolet, G. (2000). Fréchet kernels for finite-frequency traveltimes-I. Theory. Geophys. J. Int., 141(1), 157-174. doi: 10.1046/j.1365 $-246 X .2000 .00070 . x$

Delaney, E., Ermert, L., Sager, K., Kritski, A., Bussat, S., \& Fichtner, A. Passive seismic monitoring with nonstationary noise sources. GEOPHYSICS, 82(4), KS57-KS70. doi: 10.1190/geo2016-0330.1

Ermert, L., Sager, K., Afanasiev, M., Boehm, C., \& Fichtner, A. (2017). Ambient Seismic Source Inversion in a Heterogeneous Earth: Theory and Application to the Earth's Hum. J. Geophys. Res. Solid Earth, 122(11), 9184-9207. doi: 10.1002/2017JB014738

Ermert, L., Villaseñor, A., \& Fichtner, A. (2016). Cross-correlation imaging of ambient noise sources. Geophys. J. Int., 204(1), 347-364. doi: 10.1093/gji/ggv460

Fichtner, A., Stehly, L., Ermert, L., \& Boehm, C. (2017). Generalized interferometry 
I: theory for interstation correlations. Geophys. J. Int., 208(2), 603-638. doi: $10.1093 /$ gji $/$ ggw 420

Friedrich, A., Krüger, F., \& Klinge, K. (1998). Ocean-generated microseismic noise located with the Gräfenberg array. J. Seismol., 2(1), 47-64. doi: 10.1023/A: 1009788904007

Froment, B., Campillo, M., Roux, P., Gouédard, P., Verdel, A., \& Weaver, R. L.

(2010). Estimation of the effect of nonisotropically distributed energy on the apparent arrival time in correlations. GEOPHYSICS, 75(5), SA85-SA93. doi: $10.1190 / 1.3483102$

Gal, M., Reading, A. M., Ellingsen, S. P., Koper, K. D., Gibbons, S. J., \& Näsholm, S. P. (2014). Improved implementation of the $\mathrm{fk}$ and Capon methods for array analysis of seismic noise. Geophys. J. Int., 198(2), 1045-1054. doi: $10.1093 /$ gji/ggu183

Gerstoft, P., \& Tanimoto, T. (2007). A year of microseisms in southern California. Geophys. Res. Lett., 34(20), L20304. doi: 10.1029/2007GL031091

Hanasoge, S. M. (2013). The influence of noise sources on cross-correlation amplitudes. Geophys. J. Int., 192(1), 295-309. doi: 10.1093/gji/ggs015

Kimman, W. P., \& Trampert, J. (2010). Approximations in seismic interferometry and their effects on surface waves. Geophys. J. Int., 182(1), 461-476. doi: 10 $.1111 / j .1365-246 X .2010 .04632 . x$

Lehujeur, M., Vergne, J., Maggi, A., \& Schmittbuhl, J. (2017). Ambient noise tomography with non-uniform noise sources and low aperture networks: case study of deep geothermal reservoirs in northern Alsace, France. Geophys. J. Int., 208(1), 193-210. doi: 10.1093/gji/ggw373

Lin, F. C., Tsai, V. C., \& Schmandt, B. (2014). 3-D crustal structure of the western United States: Application of Rayleigh-wave ellipticity extracted from noise cross-correlations. Geophys. J. Int., 198(2), 656-670. doi: 10.1093/gji/ggu160

Liu, Q., Koper, K. D., Burlacu, R., Ni, S., Wang, F., Zou, C., ... Reading, A. M. (2016). Source locations of teleseismic P, SV, and SH waves observed in microseisms recorded by a large aperture seismic array in China. Earth Planet. Sci. Lett.. doi: 10.1016/j.epsl.2016.05.035

Löer, K., Riahi, N., \& Saenger, E. H. (2018). Three-component ambient noise beamforming in the Parkfield area. Geophys. J. Int., 213(3), 1478-1491. doi: 10 
.1093/gji/ggy058

Muir, J. B., \& Tsai, V. C. (2017). RayleighWave H/V via Noise Cross Correlation in Southern California. Bull. Seismol. Soc. Am., 107(5), 2021-2027. doi: 10.1785/ 0120170051

Nakata, N., Chang, J. P., Lawrence, J. F., \& Boué, P. (2015). Body wave extraction and tomography at Long Beach, California, with ambient-noise interferometry. J. Geophys. Res. Solid Earth, 120(2), 1159-1173. doi: 10.1002/2015JB011870

Nishida, K., \& Fukao, Y. (2007). Source distribution of Earth's background free oscillations. J. Geophys. Res., 112(B6), B06306. doi: 10.1029/2006JB004720

Pedersen, H. A., \& Krüger, F. (2007). Influence of the seismic noise characteristics on noise correlations in the Baltic shield. Geophys. J. Int., 168(1), 197210. doi: 10.1111/j.1365-246X.2006.03177.x

Roux, P. (2009). Passive seismic imaging with directive ambient noise: application to surface waves and the San Andreas Fault in Parkfield, CA. Geophys. J. Int., 179(1), 367-373. doi: 10.1111/j.1365-246X.2009.04282.x

Roux, P., \& Ben-Zion, Y. (2017). Rayleigh phase velocities in Southern California from beamforming short-duration ambient noise. Geophys. J. Int., 211(1), 450-454. doi: 10.1093/gji/ggx316

Roux, P., Sabra, K. G., Gerstoft, P., Kuperman, W. A., \& Fehler, M. C. (2005). Pwaves from cross-correlation of seismic noise. Geophys. Res. Lett., 32(19), n/an/a. doi: 10.1029/2005GL023803

Ruigrok, E., Campman, X., \& Wapenaar, K. (2011). Extraction of P-wave reflections from microseisms. Comptes Rendus Geosci., 343(8-9), 512-525. doi: 10 .1016/J.CRTE.2011.02.006

Stehly, L., \& Boué, P. (2017). On the interpretation of the amplitude decay of noise correlations computed along a line of receivers. Geophys. J. Int., 209(1), 358372. doi: $10.1093 /$ gji/ggx021

Stehly, L., Campillo, M., \& Shapiro, N. M. (2006). A study of the seismic noise from its long-range correlation properties. J. Geophys. Res., 111(B10), B10306. doi: $10.1029 / 2005 J B 004237$

Tarantola, A. (2005). Inverse Problem Theory and Methods for Model Parameter Estimation. Society for Industrial and Applied Mathematics. doi: 10.1137/1 .9780898717921 
Tromp, J., Luo, Y., Hanasoge, S., \& Peter, D. (2010). Noise cross-correlation sensitivity kernels. Geophys. J. Int., 183(2), 791-819. doi: 10.1111/j.1365-246X $.2010 .04721 . \mathrm{x}$

Tsai, V. C. (2009). On establishing the accuracy of noise tomography travel-time measurements in a realistic medium. Geophys. J. Int., 178(3), 1555-1564. doi: 10.1111/j.1365-246X.2009.04239.x

Tsai, V. C. (2011). Understanding the amplitudes of noise correlation measurements. J. Geophys. Res., 116(B9), B09311. doi: 10.1029/2011JB008483

Wang, K., Luo, Y., \& Yang, Y. (2016). Correction of phase velocity bias caused by strong directional noise sources in high-frequency ambient noise tomography: a case study in Karamay, China. Geophys. J. Int., 205(2), 715-727. doi: 10.1093/gji/ggw039

Webb, S. C. (1998). Broadband seismology and noise under the ocean. Rev. Geophys., 36(1), 105-142. doi: 10.1029/97RG02287

Yang, Y., \& Ritzwoller, M. H. (2008). Characteristics of ambient seismic noise as a source for surface wave tomography. Geochemistry, Geophys. Geosystems, 9(2), Q02008. doi: 10.1029/2007GC001814

Yao, H., \& van der Hilst, R. D. (2009). Analysis of ambient noise energy distribution and phase velocity bias in ambient noise tomography, with application to SE Tibet. Geophys. J. Int., 179(2), 1113-1132. doi: 10.1111/ j.1365-246X.2009.04329.x 